\title{
Torsion Mechanics as an Indicator of More Advanced Left Ventricular Systolic Dysfunction in Secondary Mitral Regurgitation in Patients with Dilated Cardiomyopathy: A 2D Speckle-Tracking Analysis
}

\author{
Elena Kinova Natalia Spasova Angelina Borizanova Assen Goudev \\ Cardiology Department, University Hospital "Tsaritsa Yoanna - ISUL", Sofia, Bulgaria
}

\begin{abstract}
Keywords
Dilated cardiomyopathy · Echocardiography .

Left ventricular dysfunction - Mitral regurgitation .

Left ventricular torsion · Speckle tracking
\end{abstract}

\begin{abstract}
Left ventricular (LV) twist serves as a compensatory mechanism in systolic dysfunction and its degree of reduction may reflect a more advanced stage of disease. Aim: The aim was to investigate twist alterations depending on the degree of functional mitral regurgitation (MR) by speckle-tracking echocardiography. Methods: Sixty-three patients with symptomatic dilated cardiomyopathy (DCM) were included. Patients were divided according to MR vena contracta width (VCW): group 1 with $\mathrm{VCW}<7 \mathrm{~mm}$ (mild/moderate MR) and group 2 with VCW $\geq 7 \mathrm{~mm}$ (severe MR). Results: There were no differences in LV geometry and function between groups. Group 2 showed lower endocardial basal rotation (BR) $\left(-2.04^{\circ} \pm 1.83^{\circ}\right.$ vs. $\left.-3.23^{\circ} \pm 1.83^{\circ}, p=0.012\right)$; epicardial BR $\left(-1.54^{\circ} \pm 1.18^{\circ}\right.$ vs. $\left.-2.31^{\circ} \pm 1.22^{\circ}, p=0.015\right)$; endocardial torsion $\left(0.41 \% \mathrm{~cm} \pm 0.36 \% \mathrm{~cm}\right.$ vs. $\left.0.63 \% \mathrm{~cm} \pm 0.44^{\circ} / \mathrm{cm}, p=0.033\right)$ and mid-level circumferential strain (CSmid) $(-6.12 \% \pm$ $2.64 \%$ vs. $-7.75 \% \pm 2.90 \%, p=0.028)$, when compared with group 1. Multivariable linear regression analysis identified
\end{abstract}

๑) 2018 S. Karger AG, Basel

E-Mail karger@karger.com

www.karger.com/crd endocardial BR, torsion and CSmid, as the best predictors of larger VCW. In the ROC curve analysis, endocardial BR and CSmid values greater than or equal to $-3.63^{\circ}$ and $-9.35 \%$, respectively, can differentiate patients with severe MR. Conclusions: In DCM patients, torsional profile was more altered in severe MR. Endocardial BR, endocardial torsion, and CSmid, can be used as indicators of advanced structural wall architecture damage.

(c) 2018 S. Karger AG, Basel

\section{Background}

Left ventricular (LV) remodeling in dilated cardiomyopathy (DCM) leads to leaflet tethering and annular dilatation, with the consequence of functional mitral regurgitation (MR). The best strategy for the treatment of severe chronic secondary MR is a matter for debate due to primary myocardial disease and severely depressed systolic function [1].

Recently, twist mechanics by speckle-tracking echocardiography (STE) has emerged as a new reliable quantitative tool for the assessment of LV function. Rotational and twist parameters may contribute useful comprehensive information beyond the conventional indices of $\mathrm{LV}$ 
function. DCM with altered LV geometry affects rotational mechanics. Twist serves as a compensatory mechanism in the case of systolic dysfunction, and its degree of reduction may reflect a more advanced stage of disease. In the dilated left ventricle, the fiber muscles in both layers are stretched and oriented more circumferentially, which leads to additional reduction in chamber contractility and torsion $[2,3]$. In addition to LV dilation, chronic MR would reduce LV twist even more, because of the decrease in lever arm force of the epicardial fibers [4]. Thus, twist and torsion may be a sensitive marker of remodeling of LV wall architecture, useful in the monitoring of disease progression and response to therapy. However, the importance of LV twist assessment in clinical practice still has not been established.

We hypothesized that severely reduced torsional parameters in DCM, with systolic dysfunction and secondary MR, may distinguish patients with more advanced LV remodeling from those with the potential for reverse remodeling. This can be used for guiding management and defining prognosis in these patients. The aim of this study was to assess alterations in twist mechanics depending on the degree of MR, using 2-dimensional (2D) STE.

\section{Methods}

\section{Study Population}

Prospective consecutive patients with DCM were studied, who had LV ejection fraction (EF) $<45 \%$ and end-diastolic diameter (EDD) $>58 \mathrm{~mm}$ or indexed EDD $>31 \mathrm{~mm} / \mathrm{m}^{2}$ for males and 32 $\mathrm{mm} / \mathrm{m}^{2}$ for females. All patients were NYHA functional class III and IV of heart failure. Primary MR, other significant valve lesions or valve prosthesis, atrial fibrillation, $\mathrm{LV}$ noncompaction, implanted pacemakers and devices for cardiac resynchronization therapy (CRT) or planned CRT, cardiac surgery, and a poor acoustic window were exclusion criteria. Patients with ischemic DCM who had undergone revascularization by percutaneous coronary intervention and those with coronary artery bypass grafting were ruled out. Patients technically unsuitable for speckle-tracking analysis were also excluded. Finally, 63 patients with idiopathic or ischemic DCM were included in the study. All of them received treatment with beta-blockers, diuretics, mineralocorticoid receptor antagonists, and angiotensin-converting enzyme inhibitors or angiotensin-receptor blockers, unless they were contraindicated. Before the echocardiographic examination, blood pressure was measured and body surface area was calculated.

The study was approved by our ethics committee and all patients agreed with the study protocol.

\section{Echocardiography}

The transthoracic echocardiographic examination was performed using the ultrasound machines: Philips iE33 and Philips EPIQ7, by one operator. Three consecutive cardiac cycles with a frame rate $>50 \mathrm{~Hz}$ for all views were acquired. Conventional pa- rameters were obtained according to guidelines for chamber quantification, with measurements of LV dimensions, volumes, EF (by the Simpson biplane method), and sphericity index (LV short-tolong-axis dimension ratio in end-diastolic apical 4-chamber view) [5]. Mitral filling by pulsed Doppler at the tips of the mitral leaflets and medial mitral annulus velocities by tissue Doppler imaging were measured in the apical 4-chamber view [6]. MR was assessed semiquantitatively by the measurement of vena contracta width (VCW) from the parasternal long axis and apical 4-chamber views. The color scale was set at an appropriate aliasing velocity. The narrowest part of the regurgitant jet was measured on zoom and the average value was calculated. Severe MR was defined as VCW width $\geq 7 \mathrm{~mm}$ [7].

Patients were divided into 2 groups according to the degree of MR: group 1 with mild or moderate MR and group 2 with severe MR. STE was performed according to the consensus document of the EACVI/ASE/Industry Task Force for the standardization of deformation imaging [8]. 2D views for speckle-tracking analysis were acquired from the parasternal short-axis at the level of the mitral valve, papillary muscles, and apical levels. The apical shortaxis view was obtained without visualization of the right ventricle or with the smallest right ventricular area. Apical 4-chamber, 2-chamber, and 3-chamber views were used for the measurement of longitudinal strain.

Off-line analysis of deformational and rotational LV parameters was performed by a single operator, with measurement of peak systolic values. Region of interest was adjusted to accommodate the whole thickness of the LV wall and automatic tracking with manual correction was performed where needed. Circumferential strain (CS) was measured at the basal (CSbas) and mid (CSmid) short-axis level. The software automatically divided the LV level into 6 segments. Global longitudinal strain (GLS) was calculated form 3 apical views in a 17-segmental model.

Basal rotation (BR) and apical rotation (AR) were assessed in both the endocardial and epicardial LV layers, and then used for the calculation of twist and torsion. As viewed from the apex, counterclockwise rotation is expressed as positive values and clockwise rotation as negative values. Peak BR was defined as the maximal negative value of curves from the short-axis base. AR was defined as the maximal positive values of curves on the apical short-axis view. Preserved AR was indicated by a counterclockwise positive direction and reversed rotation was defined as a negative clockwise rotation. Endocardial and epicardial LV twist was defined as the difference between the values of the peak rotations at the apical and basal level at isochronal time points. The twist is preserved when it is positive and has a normal systolic profile, and it is lost when there is negative AR and small negative BR. LV torsion or normalized twist $\left(\% / \mathrm{cm}^{2}\right)$ was defined as the twist angle divided by the distance between measured locations of the base and apex (LV twist/end-diastolic LV length) [2].

\section{Statistical Analysis}

The distribution across the groups was tested with the Kolmogorov-Smirnov test.

Normally distributed continuous variables were presented as mean \pm SD and compared using the parametric test (independentsamples $t$ test). The variables with skewed distribution were presented as medians and tested with nonparametric methods (the Mann-Whitney 2-samples test). Categorical variables were expressed as counts and percentages, and analyzed with the $\chi^{2}$ test or 
Table 1. Clinical characteristics of patients with mild/moderate and severe mitral regurgitation

\begin{tabular}{lccc}
\hline Clinical parameters & $\begin{array}{l}\text { Group 1: mild/moderate MR } \\
(n=34)\end{array}$ & $\begin{array}{l}\text { Group 2: severe MR } \\
(n=29)\end{array}$ & $p$ value \\
\hline Age, years & $60.63 \pm 13.27$ & $60.72 \pm 13.02$ & 0.852 \\
Male gender, $n$ (\%) & $25(73.5)$ & $20(69)$ & 0.783 \\
Patients with ischemic DCM, $n(\%)$ & $15(44)$ & $11(38)$ & 0.798 \\
Body surface area, ${ }^{2}$ & $1.85 \pm 0.21$ & $1.92 \pm 0.24$ & 0.256 \\
Systolic blood pressure, $\mathrm{mm} \mathrm{Hg}$ & $113.2 \pm 18.20$ & $111.36 \pm 16.63$ & 0.361 \\
Diastolic blood pressure, $\mathrm{mm} \mathrm{Hg}$ & $70.35 \pm 8.08$ & $69.70 \pm 9.23$ & 0.789 \\
Heart rate, beats/min & $72.00 \pm 20.40$ & $75.92 \pm 12.01$ & 0.267 \\
\hline
\end{tabular}

Values are expressed as mean $\pm \mathrm{SD}$, unless otherwise indicated. DCM, dilated cardiomyopathy; MR, mitral regurgitation.

Table 2. Conventional echocardiographic parameters for LV systolic and diastolic function in dilated cardiomyopathy patients with mild/moderate and severe mitral regurgitation

\begin{tabular}{llcc}
\hline Echocardiographic parameters & $\begin{array}{l}\text { Group 1: mild/moderate MR } \\
(n=34)\end{array}$ & $\begin{array}{l}\text { Group 2: severe MR } \\
(n=29)\end{array}$ & $p$ value \\
\hline End-diastolic dimension index, $\mathrm{mm} / \mathrm{m}^{2}$ & $34.43 \pm 3.76$ & $35.76 \pm 6.05$ & 0.309 \\
End-systolic dimension index, $\mathrm{mm} / \mathrm{m}^{2}$ & $28.93 \pm 6.44$ & $31.86 \pm 6.39$ & 0.078 \\
End-diastolic volume index, $\mathrm{mL} / \mathrm{m}^{2}$ & $92.00 \pm 29.71$ & $106.20 \pm 36.89$ & 0.102 \\
End-systolic volume index, $\mathrm{mL} / \mathrm{m}^{2}$ & $62.44 \pm 26.40$ & $73.81 \pm 35.90$ & 0.165 \\
Ejection fraction, \% & $33.50 \pm 7.95$ & $32.52 \pm 13.93$ & 0.745 \\
End-diastolic LV length, cm & $8.80 \pm 1.07$ & $9.1 \pm 1.11$ & 0.280 \\
End-diastolic sphericity index & $0.61 \pm 0.13$ & $0.65 \pm 0.82$ & 0.107 \\
E/A ratio & $1.84 \pm 0.93$ & $2.10 \pm 1.03$ & 0.676 \\
Sm, cm/s (range) & $4.30(2.76-9.46)$ & $3.93(2.21-8.27)$ & 0.402 \\
E'm, cm/s (range) & $4.11(2.96-12)$ & $4.17(2.51-9.57)$ & 0.176 \\
E/E'm ratio & $21.14 \pm 8.91$ & $21.74 \pm 8.04$ & 0.798 \\
\hline
\end{tabular}

Values are expressed as mean $\pm \mathrm{SD}$, unless otherwise indicated. $\mathrm{LV}$, left ventricular; $\mathrm{MR}$, mitral regurgitation; $\mathrm{E} / \mathrm{A}$ ratio, early E-wave to late A-wave ratio of diastolic mitral filling; Sm, systolic velocity of medial mitral annulus; E’m, early diastolic velocity of medial mitral annulus; E/E’m ratio, mitral E-wave to early diastolic velocity of medial mitral annulus E'm ratio.

the Fisher exact test. Bivariate correlation with the Pearson correlation coefficient was used to test the relationship between VCW and echocardiographic parameters of LV size, function, and mechanics. The best independent predictors of VCW were identified by linear stepwise regression analysis. Analysis of the area under the receiver-operating characteristics (ROC) curves was used to establish the cut-off values of the best parameters, in order to distinguish patients with severe MR.

To assess intraobserver variability and reproducibility, a second measurement of the same echocardiographic loops over time in 10 randomly chosen subjects was performed. Variability was calculated as the absolute difference between 2 sets of measurements, divided by their mean minus mean percent error. The size of measurement error was presented as standard deviation (SD) of repeated measurements on the same patient. Repeatability, representing measurement error was calculated as $(\sqrt{ } 2 \times 1.96) \times \mathrm{SD}$. To test reproducibility, the intraclass correlation coefficient (ICC) for single measures was used, with a value of 1 representing a perfect correlation. Reliability analysis with a two-way mixed-effects model and an absolute agreement type was performed.

Statistical analysis was performed with IBM SPSS for Windows v20. A two-tailed $p$ value $<0.05$ was considered statistically significant.

\section{Results}

A total of 63 patients with DCM were enrolled, 46 (72\%) were male. Mean age was $61 \pm 13$ years (range 3484 years). Thirty-four patients had idiopathic DCM and 29 had ischemic DCM. There were no statistically significant differences in age, gender, body surface area, blood 
Table 3. Echocardiographic parameters of LV deformation and torsional mechanics in patients with $\mathrm{mild} / \mathrm{mod}$ erate and severe mitral regurgitation

\begin{tabular}{|c|c|c|c|}
\hline Echocardiographic parameters & $\begin{array}{l}\text { Group 1: mild/moderate MR } \\
(n=34)\end{array}$ & $\begin{array}{l}\text { Group 2: severe MR } \\
(n=29)\end{array}$ & $p$ value \\
\hline Global longitudinal strain, $\%$ & $-8.29 \pm 3.44$ & $-6.84 \pm 2.95$ & 0.089 \\
\hline Basal circumferential strain, $\%$ & $-7.79 \pm 4.59$ & $-6.91 \pm 3.36$ & 0.400 \\
\hline Mid-level circumferential strain, \% & $-7.75 \pm 2.90$ & $-6.12 \pm 2.64$ & 0.028 \\
\hline Basal endocardial rotation, ${ }^{\circ}$ & $-3.23 \pm 1.83$ & $-2.04 \pm 1.83$ & 0.012 \\
\hline Basal epicardial rotation, ${ }^{\circ}$ & $-2.31 \pm 1.22$ & $-1.54 \pm 1.18$ & 0.015 \\
\hline Apical endocardial rotation, ${ }^{\circ}$ & $2.15 \pm 2.31$ & $1.30 \pm 2.47$ & 0.165 \\
\hline Apical epicardial rotation, ${ }^{\circ}$ & $1.63 \pm 2.12$ & $1.04 \pm 2.27$ & 0.447 \\
\hline Reversed apical rotation, $n(\%)$ & $6(17.6)$ & $10(34.5)$ & 0.154 \\
\hline Endocardial twist, ${ }^{\circ}$ & $5.56 \pm 3.59$ & $3.44 \pm 2.96$ & 0.019 \\
\hline Epicardial twist, ${ }^{\circ}$ & $3.95 \pm 2.37$ & $2.66 \pm 2.47$ & 0.043 \\
\hline Endocardial torsion, $\% \mathrm{~cm}$ & $0.63 \pm 0.44$ & $0.41 \pm 0.36$ & 0.033 \\
\hline Epicardial torsion, $\% / \mathrm{cm}$ & $0.46 \pm 0.31$ & $0.31 \pm 0.28$ & 0.050 \\
\hline
\end{tabular}

Values are expressed as mean $\pm \mathrm{SD}$, unless otherwise indicated. LV, left ventricular; MR, mitral regurgitation.

Table 4. Correlations between mitral regurgitation vena contracta width in millimeters and the indices of left ventricular size, function, and mechanics

\begin{tabular}{llc}
\hline & \multicolumn{2}{l}{$\begin{array}{l}\text { Bivariate Pearson } \\
\text { correlations }\end{array}$} \\
\cline { 2 - 3 } & Coefficient $R$ & $p$ value \\
\hline End-diastolic volume index & 0.30 & 0.017 \\
End-systolic volume index & 0.25 & 0.044 \\
Ejection fraction & -0.15 & 0.90 \\
Global longitudinal strain & 0.32 & 0.015 \\
Basal circumferential strain & 0.04 & 0.737 \\
Mid-level circumferential strain & 0.39 & 0.002 \\
Basal endocardial rotation & 0.42 & 0.001 \\
Basal epicardial rotation & 0.40 & 0.001 \\
Apical endocardial rotation & -0.28 & 0.026 \\
Apical epicardial rotation & -0.17 & 0.175 \\
Endocardial twist & -0.43 & $<0.0001$ \\
Epicardial twist & -0.36 & 0.005 \\
Endocardial torsion & -0.41 & 0.001 \\
Epicardial torsion & -0.35 & 0.005 \\
\hline
\end{tabular}

pressure, and LV indexed volumes and EF between patients with different etiologies of DCM. Patients were divided into 2 groups according to the degree of MR: group 1 with mild or moderate MR (VCW $<7 \mathrm{~mm}, n=34$ ) and group 2 with severe $\mathrm{MR}$ (VCW $\geq 7 \mathrm{~mm}, n=29)$. Clinical characteristics are described in Table 1 . There were no differences in age, gender, systolic blood pressure, and heart rate between the 2 groups.

\section{Conventional Echocardiography and STE}

Echocardiographic LV parameters are presented in Tables 2 and 3 . The $t$ test analysis showed that the 2 groups had altered LV variables of size and function, but a similar EF. In patients with severe MR, there was a tendency towards a higher end-systolic dimension index, end-diastolic and end-systolic volume indices, and GLS, but no statistical significance was reached. CS at the papillary muscle level was more significantly decreased $(-7.75 \pm$ 2.90 vs. $-6.12 \pm 2.64 \%, p=0.028)$. More severe alteration of torsional parameters with lower endocardial and epicardial twist $\left(5.56^{\circ} \pm 3.59^{\circ}\right.$ vs. $3.44^{\circ} \pm 2.96^{\circ}, p=0.019$; and $3.95^{\circ} \pm 2.37^{\circ}$ vs. $2.66^{\circ} \pm 2.47^{\circ}, p=0.043$, respectively) and endocardial and epicardial torsion $\left(0.63^{\circ} / \mathrm{cm} \pm 0.44^{\circ} / \mathrm{cm}\right.$ vs. $0.41 \% \mathrm{~cm} \pm 0.36 \% \mathrm{~cm}, p=0.033$; and $0.46^{\circ} / \mathrm{cm} \pm 0.31 \%$ $\mathrm{cm}$ vs. $0.31 \% \mathrm{~cm} \pm 0.28 \% \mathrm{~cm}, p=0.050$, respectively) were observed. Endocardial BR $\left(-3.23^{\circ} \pm 1.83^{\circ}\right.$ vs. $-2.04^{\circ} \pm$ $\left.1.83^{\circ}, p=0.012\right)$ and epicardial BR $\left(-2.31^{\circ} \pm 1.22^{\circ}\right.$ vs. $\left.-1.54^{\circ} \pm 1.18^{\circ}, p=0.015\right)$ were more significantly depressed but AR did not reach statistical significance. Reversed $A R$ was observed in 16 patients, 10 of whom (34.5\%, $p=0.15)$ had severe MR. When patients with reversed AR were excluded from the analysis, only endocardial twist remained significantly decreased in the group with severe $\operatorname{MR}\left(6.27^{\circ} \pm 2.95^{\circ}\right.$ vs. $\left.4.69^{\circ} \pm 2.29^{\circ}, p=0.048\right)$.

Correlated Factors and Predictors of the Severity of $M R$

Pearson correlations between VCW and LV indexed volumes, EF, GLS and rotational and torsional parameters were presented in Table 4. All parameters showed statisti- 
Fig. 1. a Scatterplot of the Pearson correlation between mitral regurgitation (MR) vena contracta width and left ventricular (LV) endocardial basal rotation (BR). b 2D speckle-tracking echocardiography in the short-axis view at the LV basal level. Endocardial BR $-4.08^{\circ}$ in a patient with moderate MR with a vena contracta width of $5 \mathrm{~mm}$ and $\mathrm{EF}$ of $30 \%$. c Endocardial BR $-3.37^{\circ}$ in a patient with severe MR with a vena contracta width of $8 \mathrm{~mm}$ and $\mathrm{EF}$ of $33 \%$.

LV Torsion Mechanics in Patients with DCM and Severe MR
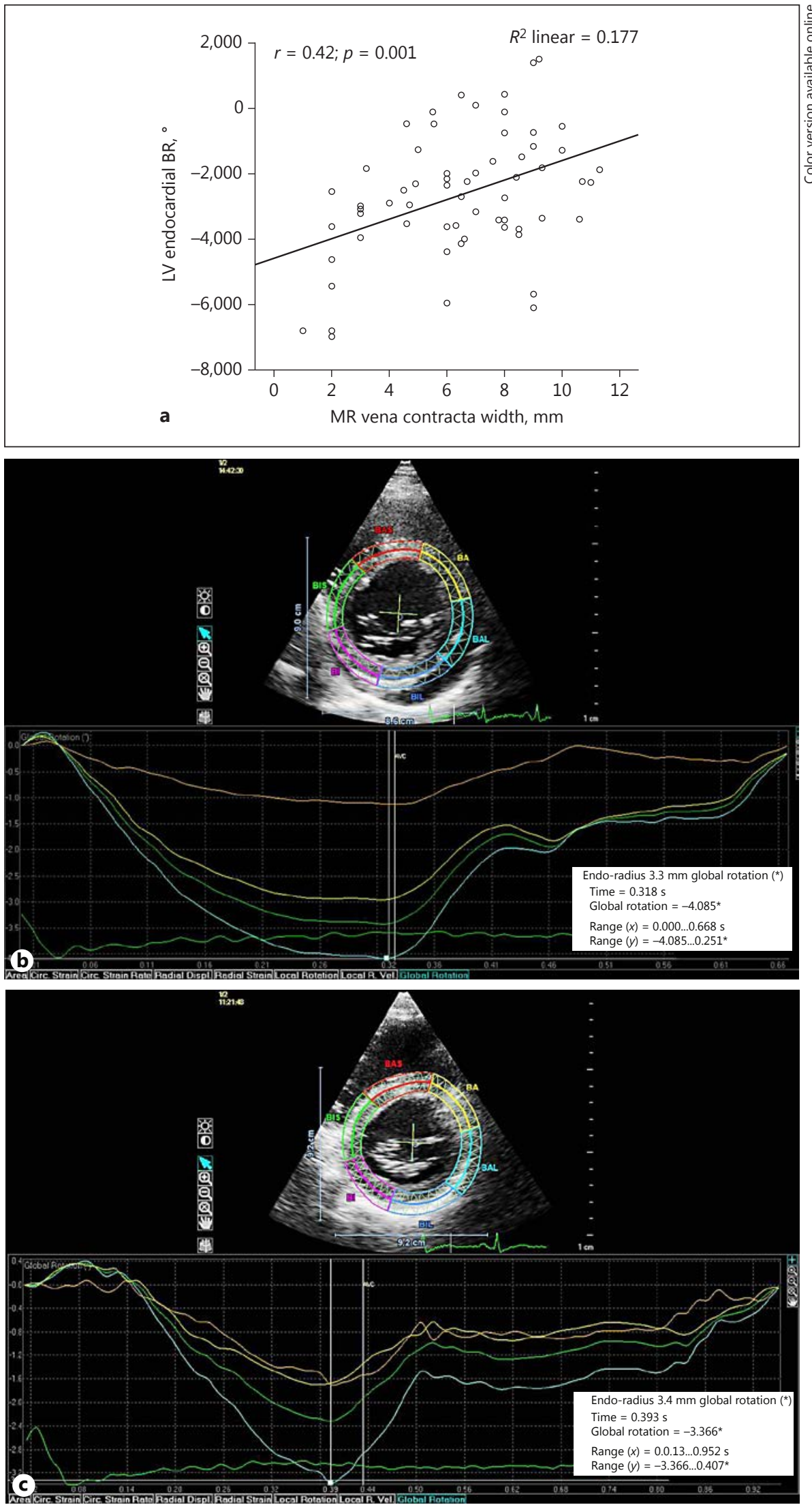

Cardiology 2018;139:187-196 


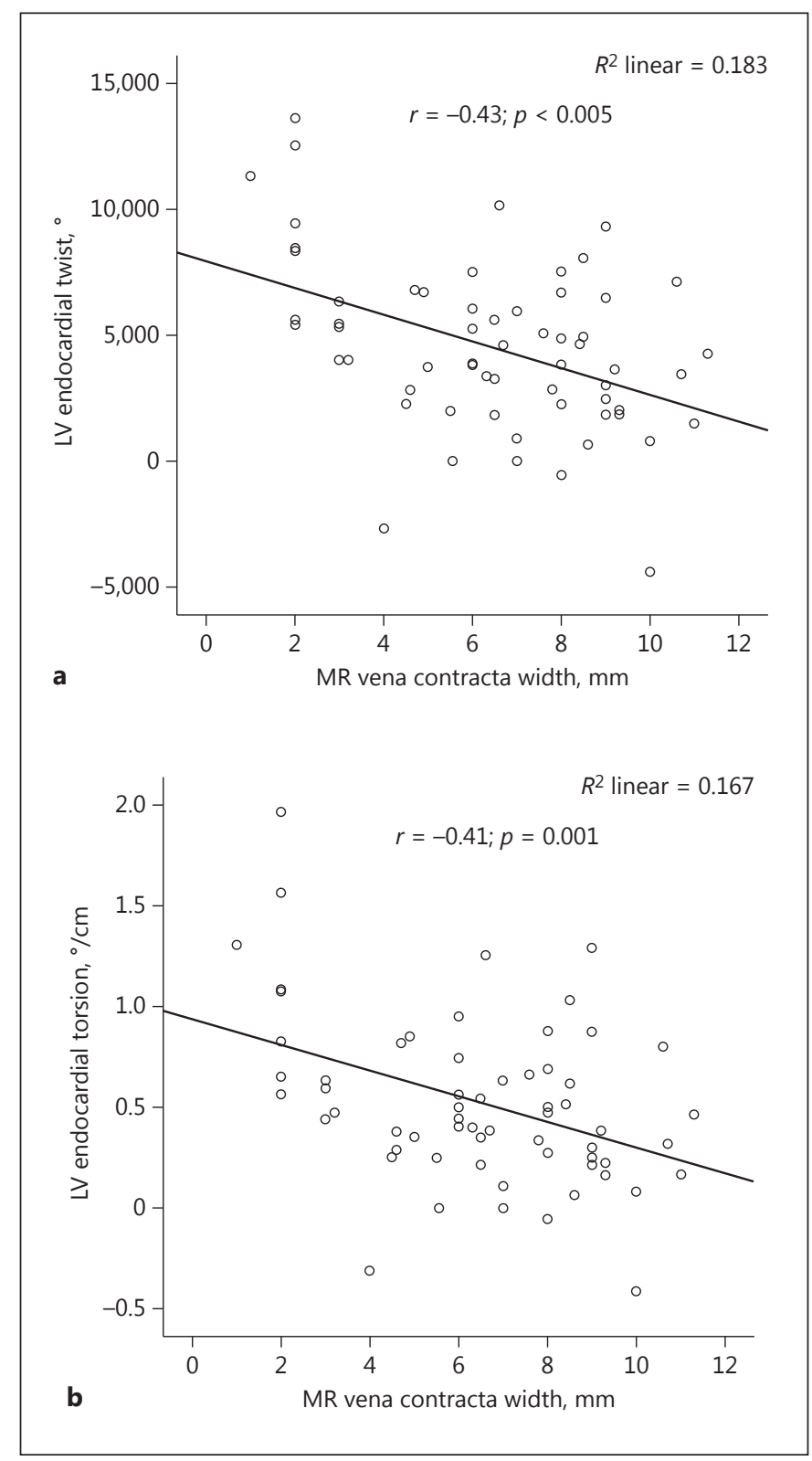

Fig. 2. Scatterplots of Pearson correlations between mitral regurgitation vena contracta width and endocardial twist (a) and torsion (b).

cally significant relationships except EF, CSbas, and epicardial AR. Moderate correlations with VCW were identified for endocardial BR $(r=0.42, p=0.001$, Fig. 1a-c), epicardial BR $(r=0.40, p=0.001)$, endocardial twist $(r=-0.43$, $p<0.005$, Fig. 2a) and endocardial torsion $(r=-0.41, p=$ 0.001 , Fig. 2b). Global longitudinal strain $(r=0.32, p=$ $0.015)$ and CSmid $(r=0.39, p=0.002)$ also showed moderate correlations which were positive. Lower negative values for BR, GLS, and CS indicate better LV function.

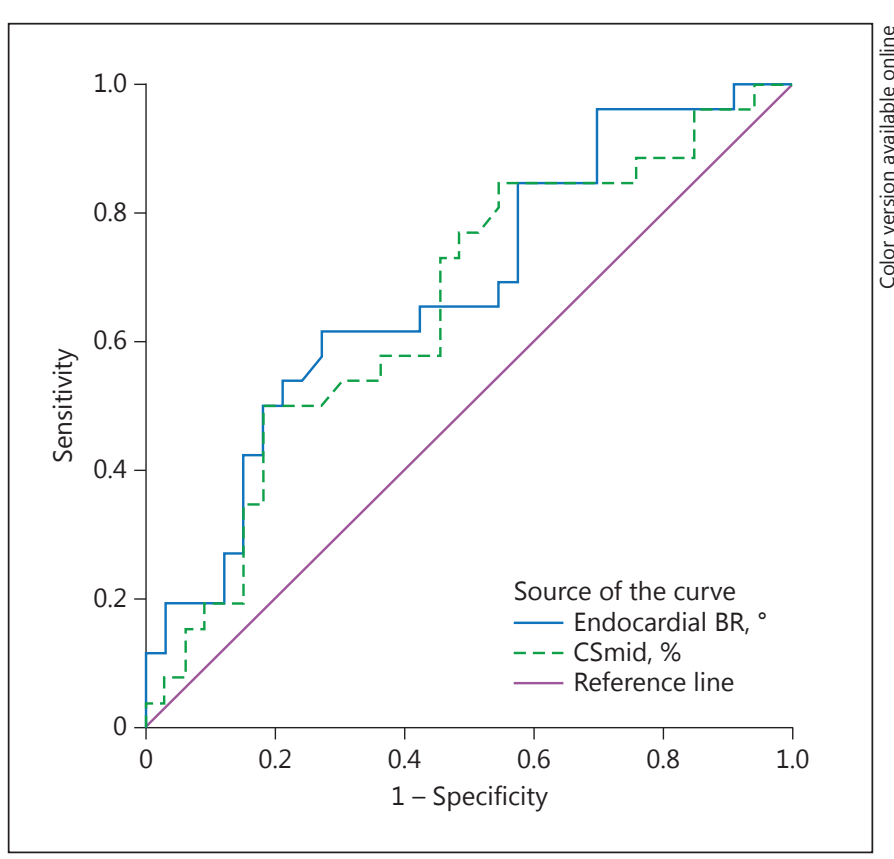

Fig. 3. The ROC curves for left ventricular endocardial basal rotation (BR; cut-off value $-3.63^{\circ}$, AUC $0.68, p=0.018$ ) and mid-level circumferential strain (CSmid; cut-off value $-9.35 \%$, AUC 0.65 , $p=0.04$ ) to identify dilated cardiomyopathy patients with severe mitral regurgitation.

These parameters were tested in multivariable linear regression analysis. The best predictive model of more severe MR, based on VCW included: endocardial BR ( $\mathrm{B}=0.53,95 \% \mathrm{CI} 0.19-0.86, p=0.003)$; endocardial torsion $(\mathrm{B}=-0.76,95 \% \mathrm{CI}-1.32$ to $-0.20, p=0.0009)$; CSmid ( $\mathrm{B}=0.24,95 \%$ CI $0.02-0.45, p=0.03)$.

ROC curve analysis showed that LV endocardial BR greater than or equal to $-3.63^{\circ}$, with an area under the curve (AUC) of $0.68(p=0.018)$, identified severe MR (95\% CI $0.54-0.82$ ) with a sensitivity of $85 \%$ and specificity of $70 \%$. The CSmid cut-off value of $-9.35 \%$ distinguished patients with severe MR (95\% CI 0.51-0.0.79) with an AUC of $0.65(p=0.04)$ with a sensitivity of $85 \%$ and a specificity of $73 \%$ (Fig. 3).

Variables of intraobserver variability, repeatability, and reproducibility of rotational parameters used for deriving twist $\left(\mathrm{AR}^{\circ}-\mathrm{BR}^{\circ}\right)$ and torsion (twist ${ }^{\circ} / \mathrm{LV}$ length, $\mathrm{cm}$ ) are presented in Table 5. The SD over 2 measurements was $0.40^{\circ}$ for endocardial twist and $0.47^{\circ}$ for epicardial twist. Repeatability was good for all parameters and indicated that, in $95 \%$ of repeated cases, the deviation of the second measure will be less than the repeatability value. High ICC between 2 measurements showed 
Table 5. Intraobserver variability, repeatability, and reproducibility of rotational parameters

\begin{tabular}{|c|c|c|c|c|c|}
\hline & Mean, ${ }^{\circ}$ & Standard deviation, ${ }^{\circ}$ & Variability & Repeatibility, $^{\circ}$ & ICC (95\% CI) \\
\hline Endocardial twist & $3.307 \pm 1.797$ & 0.40 & -0.27 & 1.11 & $0.93(0.76-0.98)$ \\
\hline Epicardial twist & $2.772 \pm 1.615$ & 0.47 & 0.26 & 1.31 & $0.88(0.60-0.97)$ \\
\hline Epicardial basal rotation & $-1.73 \pm 1.03$ & 0.31 & 0.24 & 0.87 & $0.86(0.54-0.96)$ \\
\hline Endocardial apical rotation & $0.65 \pm 1.79$ & 0.44 & 1.47 & 1.01 & $0.91(0.70-0.98)$ \\
\hline Epicardial apical rotation & $1.04 \pm 1.87$ & 0.21 & 0.03 & 0.59 & $0.98(0.93-0.99)$ \\
\hline
\end{tabular}

good agreement between different measures. Epicardial $\mathrm{AR}$, endocardial BR, endocardial twist, and endocardial AR showed an excellent level of reliability, with a very high ICC, $>0.90$. The reliability of epicardial twist and epicardial BR was good-to-excellent because the upper limit of the $95 \%$ CI was $>0.90$ (i.e., 0.97 and 0.96 , respectively).

\section{Discussion}

This study demonstrated that LV BR, twist, and torsion were significantly more reduced in patients with severe MR, despite similar LV size, geometry, and EF. LV endocardial BR, endocardial torsion, and CSmid were the best correlates of severe MR.

Changes in LV mechanics in DCM have been wellstudied. The value of LV twist may encourage the implementation of LV twist parameters in the "diagnostic toolbox" for cardiomyopathies [9]. Twist and torsion may add additional important information about LV function to conventional echocardiographic parameters.

Based on these data, we studied torsional mechanics in patients with DCM and different degrees of MR and hypothesized that patients with more severe MR would have more attenuated twist and torsion. Our DCM patients had very decreased parameters of twist mechanics and $\mathrm{BR}$, twist, and torsion were even more reduced in the group with severe MR. Severe alterations in torsional mechanics in DCM patients in comparison to healthy individuals have been reported. Decreased endocardial circumferential-longitudinal shear, which represents LV torsion, was found using magnetic resonance imaging $[10,11]$. Magnetic resonance imaging with tissue tagging is accepted as a gold standard, since it is able to create noninvasive markers over the entire myocardium [12, 13]. Echocardiographic measurement of rotation and twist in healthy persons by Philips machines was reported

LV Torsion Mechanics in Patients with DCM and Severe MR
$[14,15]$. The mean values were: $\mathrm{BR}-3.4^{\circ} \pm 0.6^{\circ}$, AR $6.6^{\circ}$ $\pm 1.9^{\circ}$, and twist $10.02^{\circ} \pm 2.1^{\circ}$ [15].

Comparison of the groups with mild/moderate and severe MR did not reveal statistically significant differences in LV size, volumes, EF and sphericity index, although the severe-MR group had slightly increased LV volumes. Deformational and torsional parameters appeared to be more sensitive markers for LV function. Patients with severe MR had more altered $B R$ and $L V$ twist and torsion but had no significant differences in AR. AR was reduced to approximately the same extent in both groups, but the base rotated less in severe MR. Reversed AR was found in $10 / 29$ patients from group 2 and in $6 / 34$ patients from group 1. After excluding these patients from the analysis, the difference in AR between the 2 groups did not change. Reversed AR was identified in cases with severe LV remodeling [16], which could be explained by a higher LV sphericity with consequent widening of the apex. Apical fiber orientation changes in a similar manner to the fibers in the basal part of the ventricle. Ascending and descending apical loop fibers lose their oblique arrangement at around $60^{\circ}$ and become more transverse up to approximately $90^{\circ}$.

Since twist represents the net difference between AR and $B R$, the more decreased $B R$ in patients with severe $\mathrm{MR}$ and the similarly reduced AR in the 2 groups, led to lower twist in group 1. Torsion represents the ratio of twist-to-LV length, and due to a lack of significant difference in LV length, torsion was also much reduced in group 1 patients. We found LV endocardial BR, endocardial torsion, and CSmid to be strong predictors for MR VCW $\geq 7 \mathrm{~mm}$. Cut-off values of endocardial BR and CSmid greater than or equal to $-3.63^{\circ}$ and $-9.35 \%$, respectively, may identify patients with severe MR.

$L V$ rotational mechanics in MR has been investigated predominantly in patients with functional MR in the setting of acute myocardial infarction, [17] primary MR for the identification of subclinical LV dysfunction, or for the 
prediction of postoperative LV function [18]. However, the data regarding LV twist mechanics in secondary MR due to DCM are sparse. The value of LV rotational parameters as well as of twist, lies in the additional information about the degree to which LV architecture and function are impaired, independently of similar LV size, geometry, and systolic and diastolic function. Recently, Notomi et al. [19] tested whether the preservation of LV torsion, defined as the net difference between the rotation angles of the apical and basal levels, is a prognostic indicator of outcome after mitral surgery for MR in patients with DCM.

It is debatable which strategy for the treatment of severe functional MR is best when there is primary myocardial disease and severe systolic dysfunction. In symptomatic patients with severe $\mathrm{MR}$ and $\mathrm{EF}>30 \%$, surgery is indicated when coronary artery bypass grafting is to be performed (NYHA class I, level C) and should be considered for patients with $\mathrm{EF}<30 \%$ when an option for revascularization exists (NYHA class IIa, level C), or when EF is $>30 \%$ with no need of revascularization but there is low comorbidity (NYHA class IIb, level C) [1]. According to the 2017 AHA/ACC focused update of the guidelines for the management of patients with valvular heart disease, valve surgery is recommended for severe secondary MR with persistent NYHA class III-IV or IIb symptoms. Now, for the first time, the preference of chordal-sparing valve replacement over annuloplasty repair is proposed in such cases (NYHA class IIa) [20].

The prognosis after mitral surgery depends on the effect of volume unloading, i.e., the "reverse remodeling viability" [21]. A higher LV sphericity index, more altered systolic torsion, and a greater degree of myocardial fibrosis represent more limited reverse remodeling viability. The degree of LV contractile reserve assessed by inotropic stimulation in chronic symptomatic heart failure, [22] may play a part in the recommendations for mitral valve surgery. The lack of response to CRT may also be an indirect marker of limited contractile reserve or remodeling viability [23].

In a series of 50 symptomatic patients with severe secondary MR in nonischemic DCM, 19 died during the follow-up period of 2.5 years [19]. Baseline LV size and shape were not statistically significant different and EF was identical in survivors and nonsurvivors, but LV torsion was higher in survivors. Assessing LV torsional mechanics may have prognostic information for the outcome of mitral valve surgery. This study demonstrated that the loss of torsion reflects irreversible structural heart damage, which makes reversed remodeling impossible after surgery. This conclusion may expand the prognosis after mitral valve surgery beyond LV improvement. Being able to identify loss of torsion may predict which patients will not benefit from surgery and which will improve with medical treatment.

Analysis of the interaction between MR and LV twist has shown that MR results from LV geometric and structural remodeling, with alterations of the mitral annulus and valvular and subvalvular apparatus. Mitral orifice and LV characteristics such as longitudinal contractility and asynchrony were identified as determining factors of functional MR [24]. In contrast, MR itself causes torsional alterations. Torsion expresses the balance between the epicardial and endocardial layers and participates in the production of a more homogenous transmural distribution of stress and fiber strain in the systole $[25,26]$. In the case of impaired torsion, the distribution of stress and strain becomes heterogeneous and leads to LV remodeling. Thus, a vicious cycle is generated and "MR begets MR". In clinical practice, the final wall architectural alterations can be assessed by rotation and twist using STE.

Measurement of LV longitudinal strain is recommended as part of echocardiographic examination, but torsional mechanics is not yet integrated in clinical practice $[8,27]$. Our intraobserver reproducibility for rotational parameters was very good, in agreement with the results of other studies. The method of tracking endocardial and epicardial points showed repeatability of endocardial and epicardial twist, i.e., $1.11^{\circ}$ and $1.31^{\circ}$, respectively. Van Dalen et al. [28] reported repeatability of twist by speckle tracking on a Philips iE33 machine 2.3. They also had good interobserver reproducibility, with the conclusion that studying changes in LV rotation over time is possible. The ICC values for both endocardial and epicardial twist in our study indicated excellent reliability, demonstrating that one operator using the same vendor facilitates an accurate assessment of torsional mechanics in patients.

\section{Study Limitations}

Our results have to be considered within the context of several limitations. The study population was small and came from a single center. There was no control group of healthy individuals, but we used reference values from other 2D STE studies on the Philips machine to show that LV strain and torsional mechanics in DCM were very depressed. The width of QRS-complex was not analyzed. There were no patients with left bundle branch block, and the sample consisted of patients for whom 
CRT devices were not suitable. For assessment of the degree of MR, the semiquantitative method for the vena contracta was used. However, there are data that the PISA method underestimates the severity of MR due to a crescent form of regurgitant orifice [7, 29]. Additionally, the measurement of VCW is more convenient and a faster method in routine clinical practice. Data on patients' adverse events, worsening heart failure, mitral valve surgery, and cardiovascular and all-cause mortality rates were not collected. These would be very important for prognostic outcome purposes.

\section{Conclusion}

In symptomatic NYHA III-IV class patients with DCM, the torsional profile was altered to a higher degree in cases with severe MR. LV endocardial BR, endocardial torsion, and CSmid may be used as markers of more advanced structural wall damage.

\section{Conflict of Interest}

There were no conflicts of interest.

\section{References}

1 Baumgartner H, Falk V, Bax J, De Bonis M, Hamm C, Holm PJ, Iung B, Lancellotti P, Lansac E, Muňoz DR, Rozenhek R, Sjögren J, Mas PT, Vahanian A, Walther T, Wendler O, Windecker S, Zamorano JL: 2017 ESC/ EACTS guidelines for the management of valvular heart disease. The Task Force for the Management of Valvular Heart Disease of the European Society of Cardiology (ESC) and the European Association for Cardio-Thoracic Surgery (EACTS). Eur Heart J 2017;00:153.

2 Sengupta P, Tajik J, Chandrasekaran K, Khanderia B: Twist mechanics of the left ventricle. Principles and application. JACC Cardiovasc Imaging 2008;1:366-376.

3 Omar AMS, Vallabhajosyula S, Sengupta P: Left ventricular twist and torsion. Research observations and clinical applications. Circ Cardiovasc Imaging 2015;8:e003029.

4 Tibayan F, Rodriguez F, Langer F, Zasio MK, Bailey L, Liang D, Daughters G, Ingels $\mathrm{N}$, Miller C: Alterations in left ventricular torsion and diastolic recoil after myocardial infarction with and without chronic ischemic mitral regurgitation. Circulation 2004; 110(suppl II):II-109-II-114.

5 Lang R, Badano L, Mor-Avi V, Afilalo J, Armstrong A, Ernande L, Flachskampf F, Foster E, Goldstein S, Kuznetsova T, Lancellotti P, Muraru D, Picard M, Rietzschel E, Rudski L, Spencer K, Tsang W, Voigt JU: Recommendations for cardiac chamber quantification by echocardiography in adults: an update from the American Society of Echocardiography and the European Association of Cardiovascular Imaging. Eur Heart J 2015;16:233-271.

6 Nagueh S, Smiseth O, Appleton C, Byrd B, Dokanish H, Edvardsen T, Flachskampf F, Gillebert T, Klein A, Lanchellotti P, Marino P, Oh J, Popescu B, Waggoner A: Recommendations for the evaluation of left ventricular dia- stolic function by echocardiography: an update from the American Society of Echocardiography and the European Association of Cardiovascular Imaging. J Am Soc Echocardiogr 2016;29:277-314.

7 Lanchellotti P, Tribouilloy C, Hagendorff A, Popescu B, Edvarsen T, Pierard L, Badano L, Zamorano J; Scientific Document Committee of the European Association of Cardiovascular Imaging: Recommendations for the echocardiographic assessment of native valvular regurgitation: an executive summary from the European Association of Cardiovascular Imaging. Eur Heart J 2013;14:611-644.

8 Voigt JU, Pedrizzetti G, Lysyansky P, Marwick T, Houle H, Baumann R, Pedri S, Ito Y, Abe Y, Metz S, Song J, Hamilton J, Sengupta P, Kolias T, d'Hooge J, Aurigemma G, Thomas J, Badano L: Definitions for a common standard for 2D speckle tracking echocardiography: consensus document of the EACVI/ASE/Industry Task Force to standardize deformation imaging. Eur Heart J 2015;16:1-11.

9 Kauer F, Geleijnse ML, Van Dalen BM: Role of left ventricular twist mechanics in cardiomyopathies, dance of the helices. World J Cardiol 2015;7:476-482.

10 Rüssel I, Götte M, Bronzwaer J, Knaapen P, Paulus W, van Rossum A: Left ventricular torsion: an expanding role in the analysis of myocardial dysfunction. J Am Coll Cardiol Img 2009;2:648-655.

11 MacGowan G, Shapiro E, Azhari H, Siu C, Hees P, Hutchins G, Weiss J, Rademakers F: Noninvasive measurement of shortening in the fiber and cross-fiber directions in the normal human left ventricle and in idiopathic dilated cardiomyopathy. Circulation 1997;96: 535-541.

12 Axel L, Dougherty L: MR imaging of motion with spatial modulation of magnetization. Radiology 1989;171:841-845.
13 Zerhouni EA, Parish DM, Rogers WJ, Yang A, Shapiro EP: Human heart: tagging with MRimaging. Radiology 1988;169:59-63.

14 Zhang HJ, Wang H, Sun T, Lu MJ, Xu N, Wu WC, Sun X, Wang WG, Lin QW: Assessment of left ventricular twist mechanics by speckle tracking echocardiography reveals association between LV twist and myocardial fibrosis in patients with hypertrophic cardiomyopathy. Int J Cardiovasc Imaging 2014;30:15391548.

15 Peters F, Khandheria B, Libhaber E, Maharaj N, dos Santos C, Matioda H, Essop M: Left ventricular twist in left ventricular noncompaction. Eur Heart J 2014;15:48-55.

16 Popescu B, Beladan C, Călin A, Muraru D, Deleanu D, Roşca M, Ginghina: Left ventricular remodelling and torsional dynamics in dilated cardiomyopathy: reversed apical rotation as a marker of disease severity. Eur J Heart Fail 2009;11:945-951.

17 Valuckiene Z, Ovsianas J, AblonskyteDudoniene R, Mizariene V, Melinyte K, Jurkevicius R: Left ventricular mechanics in functional ischemic mitral regurgitation in acute inferoposterior myocardial infarction. Echocardiography 2016;33:1131-1142.

18 Candan O, Hatipoglu Akpinar S, Dogan C, Demirkiran A, Dinbar B, Bayram Z, Yilmaz F, Kaymaz C, Ozdemir N: Twist deformation for predicting postoperative left ventricular function in patients with mitral regurgitation: a speckle tracking echocardiography study. Echocardiography 2017;34:422-428.

19 Notomi Y, Isomura T, Kanai S, Maeda M, Hoshino J, Kondo T, Fukada Y, Furukawa K: Pre-operative left ventricular torsion, QRS width/CRT, and post-mitral surgery outcomes in patients with nonischemic, chronic, severe secondary mitral regurgitation. J Am Coll Cardiol Basic Trans Sci 2016;1: 193-202. 
20 Nishimura R, Otto C, Bonow R, Carabello B, Erwin J, Fleisher L, Jneid H, Mack M, McLeod C, O'Gara P, Rigolin V, Sundt T, Thompson A: 2017 AHA/ACC focused update of the 2014 AHA/ACC guideline for the management of patients with valvular heart disease. A report of the American College of Cardiology/American Heart Association Task Force on clinical practice guidelines. Developed in collaboration with the American Association for Thoracic Surgery, American Society of Echocardiography, Society for Cardiovascular Angiography and Interventions, Society of Cardiovascular Anesthesiologists, and Society of Thoracic Surgeons. Circulation 2017;135:e1159-e1195.

21 Di Salvo T, Acker M, Dec GW, Byrne J: Mitral valve surgery in advanced heart failure. J Am Coll Cardiol 2010;55:271-282.

22 Piechota W, Gielerak G: The prognostic value of stress tests in chronic heart failure. Cardiol J 2007;14:340-346.
23 Ypenburg C, van Bommel RJ, Borleffs CJ: Long-term prognosis after cardiac resynchronization therapy is related to the extent of left ventricular reverse remodeling at midterm follow-up. J Am Coll Cardiol 2009;53:483490.

24 Donal E, de Place C, Kervio G, Bauer F, Gervais R, Leclercq C, Mabo Ph, Daubert JC: Mitral regurgitation in dilated cardiomyopathy: value of both regional left ventricular contractility and dyssynchrony. Eur J Echocardiogr 2009;10:133-138.

25 Ennis D, Nguyen T, Itoh A, Bothe W, Liang D, Ingels N, Miller C: Reduced systolic torsion in chronic "pure" mitral regurgitation. Circ Cardiovasc Imaging 2009;2:85-92.

26 Bovendeerd P, Arts T, Huyghe J, van Campen D, Reneman R: Dependence of local left ventricular wall mechanics on myocardial fiber orientation: a model study. J Biomech 1992; 25:1129-1140.
27 Galderisi M, Cosyns B, Edvardsen T, Cardim N, Delgado V, Di Salvo G, Donal E, Sade LE, Ernande L, Gabri M, Grapsa J, Hagendorff A, Kamp O, Magne J, Santoro C, Stefanidis A, Lancellotti P, Popescu B, Habib G: Standardization of adult transthoracic echocardiography reporting in agreement with recent chamber quantification, diastolic function, and heart valve disease recommendations: an expert consensus document of the European Association of Cardiovascular Imaging. Eur Heart J 2017;0:1-10.

28 Van Dalen B, Soliman O, Vletter W, Kauer F, van der Zwaan $\mathrm{H}$, ten Cate F, Geleijnse M: Feasibility and reproducibility of left ventricular rotation parameters measured by speckle tracking echocardiography. Eur J Echocardiogr 2009;10:669-676.

29 Uretsky S, Gillam L, Lang R, Chaudhry F, Argulian E, Supariwala A, Gurram S, Jain K, Subero M, Jang J, Cohen R, Wolff S: Discordance between echocardiography and MRI in the assessment of mitral regurgitation severity: a prospective multicenter trial. J Am Coll Cardiol 2015;65:1078-1088. 